Electrostatic coupling and interface intermixing in ferroelectric superlattices

This article has been downloaded from IOPscience. Please scroll down to see the full text article.

2012 EPL 9946004

(http://iopscience.iop.org/0295-5075/99/4/46004)

View the table of contents for this issue, or go to the journal homepage for more

Download details:

IP Address: 202.185.114.7

The article was downloaded on 15/07/2013 at 03:33

Please note that terms and conditions apply. 


\title{
Electrostatic coupling and interface intermixing in ferroelectric superlattices
}

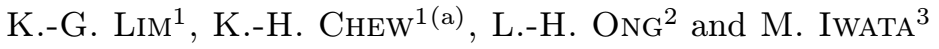 \\ 1 Department of Physics, University of Malaya - 50603 Kuala Lumpur, Malaysia \\ 2 School of Physics, Universiti Sains Malaysia - 11800 Minden, Penang, Malaysia \\ 3 Department of Engineering Physics, Electronics and Mechanics, Graduate School of Engineering, \\ Nagoya Institute of Technology - Nagoya 466-8555, Japan
}

received 23 May 2012; accepted in final form 30 July 2012

published online 29 August 2012

PACS 68.65.Cd - Superlattices

PACS 77.22.Ej - Polarization and depolarization

PACS 77.80.bn - Strain and interface effects

\begin{abstract}
A thermodynamic model is developed to study electrostatic coupling and interface intermixing in superlattices comprising alternate layers of ferroelectrics and paraelectrics. Interface intermixing leads to inhomogeneous internal electric field and polarization in superlattices. The spatial distribution of polarization extends into the layer over a distance governed by its correlation length. Periodic modulation of the internal electric field and polarization in superlattices are correlated. Interface intermixing enhances the depolarization field of superlattices; however, it has a negligible effect on polarization and transition temperature. The internal electric field, originating from the electrostatic coupling between ferroelectric layers, plays a dominant role in determining the properties of superlattices.
\end{abstract}

Copyright (c) EPLA, 2012

Ferroelectric superlattices composed of alternating layers are currently a topic of active research due to their potential applications $[1,2]$, as well as their striking new behaviors [3]. In a superlattice system, intermixed layers may form at interfaces between two layers. Although superlattices of $\mathrm{ABO}_{3}$ perovskite oxides such as $\mathrm{BaTiO}_{3} / \mathrm{SrTiO}_{3}$ superlattices can be fabricated without interface intermixing [4], intermixing at interfaces is usually difficult to control experimentally at high temperature using high-energy lasers, where the stoichiometry of the deposited films changes in a complicated manner under the prescribed deposition conditions $[5,6]$. These intermixed layers at interfaces, with properties different from those of both layers, may affect the properties of superlattices.

The formation of intermixed layers can be induced by short-range interactions between materials in close contact, surface or interface reconstruction, cation intermixing or composition deviations at the interfaces in superlattices of ferroelectric solid solution [7]. Fong et al. [8] studied the interface structure of $\mathrm{PbTiO}_{3}$ thin films grown on $\mathrm{SrTiO}_{3}$ substrates by a high-resolution

(a) E-mail: khchew@um.edu.my coherence Bragg rod analysis (COBRA). Their work indicates that cation intermixing may be present at interfaces of $\mathrm{PbTiO}_{3} / \mathrm{SrTiO}_{3}$ superlattices. Hung et al. [9] and Ishibashi et al. [10] demonstrated the existence of compositional intermixed layers at the interface of $\mathrm{PbZrO}_{3} /$ $\mathrm{BaZrO}_{3}$ superlattices.

Cooper and co-workers [11] studied the effect of interface intermixing in short-period $\mathrm{PbTiO}_{3} / \mathrm{SrTiO}_{3}$ superlattices by the first-principle density functional theory, and showed that interface intermixing can significantly enhance the ferroelectric properties of the superlattice. A study on the structural evolution of surfaces during the layer-by-layer growth of $\mathrm{BaTiO}_{3}$ films on $\mathrm{SrRuO}_{3}$ indicates that the surface reconstruction of $\mathrm{SrRuO}_{3}$ increases the oxygen concentration, and leads to both intermixing and a structural change in $\mathrm{BaTiO}_{3}$ at the interface [12]. Their finding reveals the possible existence of an intermixed layer at the oxide interface. Recently, Mizoguchi and co-workers [5] demonstrated the possibility of improving the properties of $\mathrm{SrTiO}_{3}$-based superlattices by controlling the interface intermixing.

Various thermodynamic models have been proposed to account for the properties of ferroelectric superlattices [13-19]. Those works, however, do not consider the 
interface intermixing effect. Pertsev and Tyunina [7] have recently proposed a thermodynamic model to study the permittivity of a superlattice by introducing an interface layer with properties different from those of both layers. However, the effect of the interface is not considered, the polarization is homogeneous and the local polarization coupling at the interface is neglected.

Recently, Torres-Pardo et al. [20] studied local ferroelectric distortions in $\mathrm{PbTiO}_{3} / \mathrm{SrTiO}_{3}$ superlattices by electron-energy-loss spectra (EELS) and high-angle annular-dark-fields (HAADF) images. They have identified local structural distortion at the single unit-cell scale across the interface, and revealed the existence of an inhomogeneous polarization profile within the ferroelectric and paraelectric layers. While the length scale of inhomogeneity extending over 5-6 unit cells was attributed to ferroelectric domains, the effect of intermixing confined to 1 unit cell from the interface may also be present and should not be ruled out. Their interesting observations indicate that the homogeneous polarization model $[7,14,15,21,22]$ does not apply to the superlattices.

We have recently proposed a thermodynamic model to study the ferroelectric properties of superlattices consisting of alternate ferroelectric and paraelectric layers [23-25]. The model can be constructed using the concept of interaction of dipole lattices, which are characterized by polarizations with double potential wells $[19,25]$. In the model, an interface energy term is introduced in the free energy to describe the local polarization coupling at the interface between the two contacting ferroelectrics, and the formation of an intermixed layer [26]. Despite its simplification, the approach has captured the essential physics which associates with polarization continuities or discontinuities, polarization inhomogeneities, intermixing and local polarization coupling at interfaces [19,23-25].

In those studies [23-25], however, the model assumes that the polarization is parallel to the surfaces or interfaces of the layered structure in which the depolarization field effect can be ignored. It is the objective of this study to investigate the case of polarization perpendicular to the surface or interface of a superlattice with the appropriate electrostatic boundary conditions. In particular, we study the effects of electrostatic coupling and interface intermixing on the internal electric field and polarization of superlattices, composed of alternate layers of ferroelectrics and paraelectrics, which can be grown on a substrate, as schematically shown in fig. 1 .

By assuming that the whole spatial variation of polarization takes place along the $z$-direction, the Helmholtz free energy per unit area for one period of the superlattice can be expressed as the follows:

$$
F=F_{F E}+F_{P E}+F_{I}
$$

The free energy per unit area of the ferroelectric layer with thickness $d_{F E}$ is $F_{F E}=\int_{-d_{F E}}^{0} f_{F E} \mathrm{~d} z$, and the free energy per unit area of the paraelectric layer with thickness $d_{P E}$

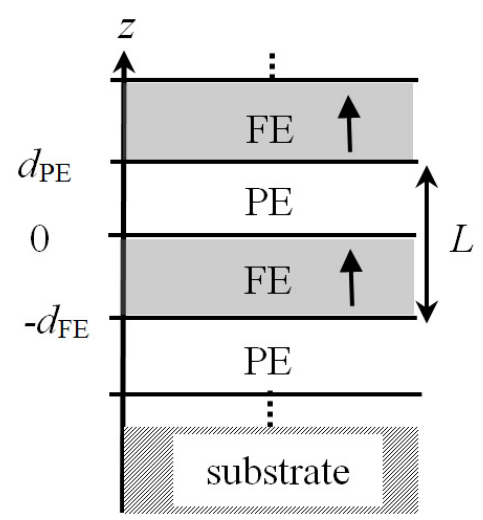

Fig. 1: Schematic illustration of a periodic superlattice composed of a ferroelectric layer and a paraelectric layer. The thicknesses of ferroelectric layer $(\mathrm{FE})$ and paraelectric layer (PE) are $d_{F E}$ and $d_{P E}$, respectively. Arrow indicates the direction of polarization.

is $F_{P E}=\int_{0}^{d_{P E}} f_{P E} \mathrm{~d} z \cdot f_{F E}$ and $f_{P E}$ are the free-energy densities, given as [27]

$$
\begin{aligned}
f_{j}= & \alpha_{j}^{*} p_{j}^{2}+\beta_{j}^{*} p_{j}^{4}+\gamma_{j} p_{j}^{6}+\frac{\kappa_{j}}{2}\left(\frac{\mathrm{d} p_{j}}{\mathrm{~d} z}\right)^{2} \\
& +\left(\frac{c_{11, j}^{2}+c_{11, j} c_{12, j}-2 c_{12, j}^{2}}{c_{11, j}}\right) u_{m, j}^{2}-\frac{1}{2} E_{d, j} p_{j} .
\end{aligned}
$$

In eq. $(2), p_{j}$ corresponds to the polarization of layer $j$ $(j: F E$ or $P E) . \alpha_{j}^{*}=\alpha_{j}+2\left(c_{12 j} g_{11 j} / c_{11 j}-g_{12 j}\right) u_{m j}$ and $\beta_{j}^{*}=\beta_{j}-g_{11 j}^{2} / 2 c_{11 j}$ where $\alpha_{j}$ (temperature dependent), $\beta_{j}$ and $\gamma_{j}$ are the Landau coefficients. $c_{11 j}$ and $c_{12 j}$ are the elastic stiffness coefficients, whereas $g_{11 j}$ and $g_{12 j}$ denote the electrostrictive constants. $u_{m j}=\left(a_{s}-a_{j}\right) / a_{s}$ denotes the in-plane misfit strain induced by the substrate due to the lattice mismatch. $a_{j}$ is the unconstrained equivalent cubic-cell lattice constant of layer $j$ and $a_{s}$ is the lattice parameter of the substrate. $\kappa_{j}$ denotes the gradient coefficient that determines the energy cost due to the inhomogeneity of polarization $p_{j} . E_{d j}$ acts as the depolarization field of layer $j$, if its direction is opposite to the direction of ferroelectric polarization. If $E_{d j}$ inclines in the same direction of polarization, it cannot be regarded as the depolarization field; thus, we denote $E_{d j}$ as "the internal electric field".

The interface energy is given by $[19,23-25]$

$$
\begin{aligned}
F_{I}= & \frac{\lambda_{0}}{2 \varepsilon_{0}}\left[\left(p_{F E}(0)-p_{P E}(0)\right)^{2}\right. \\
& \left.+\left(p_{F E}\left(-d_{F E}\right)-p_{P E}\left(d_{P E}\right)\right)^{2}\right],
\end{aligned}
$$

where $\lambda_{0}$ is the temperature-independent interface parameter and $\varepsilon_{0}$ is the dielectric permittivity in vacuum. $p_{F E}(0)$ and $p_{F E}\left(-d_{F E}\right)$ represent the polarization at the interface of the ferroelectric layer, whereas the interface polarizations of the paraelectric layer are represented by $p_{P E}(0)$ and $p_{P E}\left(d_{P E}\right)$. It is important to mention that the interface energy (3) possesses a similar form as the gradient terms in eq. (2) $[19,25]$. 
The physical origin of the interface energy (3) can be interpreted as follows [19,25]. By symmetry, we have $p_{F E}(0)=p_{F E}\left(-d_{F E}\right)=p_{i}$ and $p_{P E}=p_{P E}\left(d_{P E}\right)=q_{i}$ and thus, eq. (3) can be written as $F_{i}=\frac{\lambda_{0}}{\varepsilon_{0}}\left(p_{i}-q_{i}\right)^{2}=\frac{\lambda_{0}}{\varepsilon_{0}}\left(p_{i}^{2}+\right.$ $\left.q_{i}^{2}\right)-2 \frac{\lambda_{0}}{\varepsilon_{0}} p_{i} q_{i}$. In other words, the interface energy expression can be clearly interpreted by separating it, (3), into two parts: i) the non-ferroelectric part and ii) the polarizations coupling part. It is well known that dead layers are intrinsic and appear inevitably at the metalferroelectric interface [28]. The bonding at the metalferroelectric interfaces of ultrathin ferroelectric capacitors, which constituted the dead layers, strongly affects the properties at the interface through the formation of intrinsic dipole moments at the interface. In the present model, the former term is analogous to the formation of "dead" layers [28] at interfaces, i.e., the surfaces of layer $F E$ (" $\lambda_{0} p_{i}^{2} / \varepsilon_{0}$ ") and layer $P E\left(" \lambda_{0} q_{i}^{2} / \varepsilon_{0}\right.$ "). The dead layers are linear dielectrics, and their dielectric stiffnesses are determined by the interface parameter $\lambda_{0}>0$. The polarization coupling part " $\lambda_{0} p_{i} q_{i} / \varepsilon_{0}$ " describes the mutual interactions between the local polarization at interfaces due to the modification of bonding at the interfaces.

In the present study of superlattices, the interface parameter $\lambda_{0}$ describes the effect of interface intermixing in the superlattice as a whole. The explicit expression derived from the interface structure for the simple case without electrostatic boundary conditions indicates that the intermixed layer is governed by the physical properties of the two constituent layers [26]. The interface intermixing effects are governed by the inhomogeneity of polarization near the interface which may arise from the formation of intrinsic dipole moments and the effect of coupling between the local polarization at the interface. The continuity or discontinuity of polarizations across the interface depends upon the nature of the intermixed layer formed at interfaces. If $\lambda_{0} \neq 0$, an intermixed layer [26] (analogous to a dead layer [28]) with properties different from those of both constituent layers is expected to form at the interface region. Polarization may be induced at the interface of the paraelectric layer, depending on its dielectric stiffness. If $\lambda_{0}=0$, no intermixed layer is formed at the interface region. Therefore, the polarization in the ferroelectric layer is homogeneous and no induced polarization is expected in the paraelectric layer.

The internal electric field $E_{d j}$ within the constituent layer may be found using Maxwell's equations that are, in the present case with no free charges $[16,29]$,

$$
\begin{gathered}
\nabla \cdot\left(\varepsilon_{0} \mathbf{E}+\mathbf{P}\right)=0, \\
\nabla \times \mathbf{E}=0,
\end{gathered}
$$

where $\mathbf{E}$ is the electric field and $\mathbf{P}$ is the polarization. Equation (4b) indicates that we may define a scalar function $\varphi_{j}$, i.e., the electrostatic potential, which satisfies $E_{d, j}=-\nabla \varphi_{j}$. Using $E_{d, j}=-\nabla \varphi_{j}(j: F E$ or $P E)$, the Euler-Lagrange equations follow from eqs. (2) can be expressed in terms of the electrostatic potential $\varphi_{j}$ as

$$
\kappa_{j} \frac{\mathrm{d}^{2} p_{j}}{\mathrm{~d} z^{2}}=2 \alpha_{j}^{*} p_{j}+4 \beta_{j}^{*} p_{j}^{3}+6 \gamma_{j} p_{j}^{5}+\frac{1}{2} \frac{\mathrm{d} \varphi_{j}}{\mathrm{~d} z},
$$

where the electrostatic potentials are $[16,29]$

$$
-\varepsilon_{0} \frac{\mathrm{d}^{2} \varphi_{j}}{\mathrm{~d} z^{2}}+\frac{\mathrm{d} p_{j}}{\mathrm{~d} z}=0
$$

and can be found using Maxwell's equation (4a). At the interface, the boundary conditions for the polarization are

$$
\left\{\begin{array}{l}
-\left.\kappa_{F E} \frac{\mathrm{d} p}{\mathrm{~d} z}\right|_{z=-d_{F E}}+\frac{\lambda_{0}}{\varepsilon_{0}}\left[p_{F E}\left(-d_{F E}\right)-p_{P E}\left(d_{P E}\right)\right]=0 \\
\left.\kappa_{P E} \frac{\mathrm{d} p}{\mathrm{~d} z}\right|_{z=0}+\frac{\lambda_{0}}{\varepsilon_{0}}\left[p_{F E}(0)-p_{P E}(0)\right]=0 \\
\left.\kappa_{F E} \frac{\mathrm{d} p}{\mathrm{~d} z}\right|_{z=0}+\frac{\lambda_{0}}{\varepsilon_{0}}\left[p_{F E}(0)-p_{P E}(0)\right]=0 \\
-\left.\kappa_{P E} \frac{\mathrm{d} p}{\mathrm{~d} z}\right|_{z=d_{P E}}+\frac{\lambda_{0}}{\varepsilon_{0}}\left[p_{F E}\left(-d_{F E}\right)-p_{P E}\left(d_{P E}\right)\right]=0
\end{array}\right.
$$

For the electrostatic boundary conditions, the continuity of the electric displacement at the interface gives

$$
\left\{\begin{array}{l}
-\left.\varepsilon_{0} \frac{\mathrm{d} \varphi_{F E}}{\mathrm{~d} z}\right|_{z=0}+\left.\varepsilon_{0} \frac{\mathrm{d} \varphi_{P E}}{\mathrm{~d} z}\right|_{z=0}= \\
-\left(p_{F E}(0)-p_{P E}(0)\right), \\
-\left.\varepsilon_{0} \frac{\mathrm{d} \varphi_{F E}}{\mathrm{~d} z}\right|_{-\mathrm{d}_{F E}}+\left.\varepsilon_{0} \frac{\mathrm{d} \varphi_{P E}}{\mathrm{~d} z}\right|_{-\mathrm{d}_{F E}}= \\
-\left(p_{F E}\left(-d_{F E}\right)-p_{P E}\left(d_{P E}\right)\right),
\end{array}\right.
$$

and the continuity of the tangential component of the electric field gives the following conditions on the electric potentials:

$$
\left\{\begin{array}{l}
\varphi_{F E}=\varphi_{P E}(0), \\
\varphi_{F E}\left(-d_{F E}\right)=\varphi_{P E}\left(d_{P E}\right) .
\end{array}\right.
$$

In the present study, eqs.(5) and (6) were transformed into a system of nonlinear equations. Solving the nonlinear equations numerically, using the finite-difference method, with the boundary conditions of eqs. (7) to (9), we obtain the spatial dependence of the internal electric field (or electrostatic potential) and polarization for the superlattices.

For illustration, we apply the model to perform a numerical calculation on a superlattice consisting of a ferroelectric layer as $\mathrm{PbTiO}_{3}(\mathrm{PT})$ and a paraelectric layer as $\mathrm{SrTiO}_{3}(\mathrm{ST})$ on a $\mathrm{ST}$ substrate, as a representative system. For the convenient of discussion, we adopt the thermodynamic coefficients of the chosen materials $^{1}$ from Dawber et al. [21]. We compare the calculated polarization and transition temperature to those

\footnotetext{
${ }^{1}$ List of parameters used in calculations (SI units and $T$ in $\mathrm{K}$ ). For PT: $\alpha_{F E}=\alpha_{0 F E}\left(T-T_{0 F E}\right), \alpha_{0 F E}=3.8 \times 10^{5}, \beta_{F E}=4.229 \times$ $10^{8}, \gamma_{F E}=2.6 \times 10^{8}, T_{0 F E}=752, g_{11 F E}=1.14 \times 10^{10}, g_{12 F E}=$ $4.63 \times 10^{8} ; \quad c_{11 F E}=1.746 \times 10^{11}, \quad c_{12 F E}=0.794 \times 10^{11}, \quad \kappa_{F E}=$ $1.029 \times 10^{-10} ;$ For ST: $\alpha_{P E}=\alpha_{0 P E}\left(T-T_{0 P E}\right), \alpha_{0 P E}=7.45 \times$ $10^{5}, \beta_{P E}=2.02 \times 10^{9}, T_{0 P E}=51.64, g_{11 P E}=1.25 \times 10^{10}, g_{12 P E}=$ $-0.108 \times 10^{10} ; \quad c_{11 P E}=3.36 \times 10^{11}, \quad c_{12 P E}=1.07 \times 10^{11}, \quad \kappa_{P E}=$ $1.029 \times 10^{-10}$.
} 


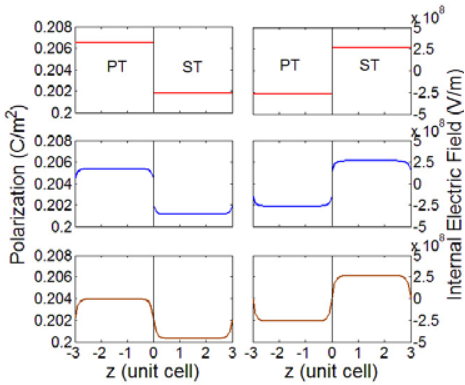

(a)

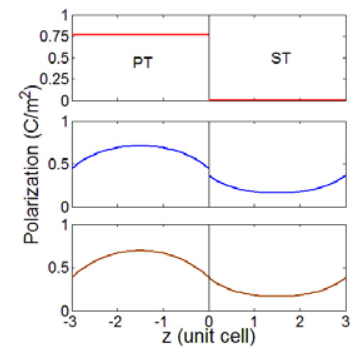

(b)

Fig. 2: (Color online) Spatial dependence of electrical properties at $T=298 \mathrm{~K}$ with electrostatic coupling (a) and without electrostatic coupling (b). The values of $\lambda_{0}$ are: 0 (red line), $0.02 \xi_{0}$ (blue line) and $\xi_{0}$ (brown line).

obtained in experiments [21]. In the calculations, we take 1 unit cell (u.c.) $\approx 0.4 \mathrm{~nm}[30]$ and the thickness of the ST layer is maintained at $d_{P E} \approx 3$ u.c. The characteristic length, $\xi_{0}=\sqrt{\kappa_{F E} /\left(\alpha_{0 F E} T_{0 F E}\right)} \sim 0.6 \mathrm{~nm}$, corresponds to the estimated length of the domain wall half-width $[16,30]$. The lattice constants in the cubic phase are $a_{F E}=3.969 \AA$ and $a_{P E}=3.905 \AA$ for PT and ST, respectively [21]. Based on the lattice constants, the lattice misfit strains in the $\mathrm{PT}$ and ST layers are obtained as $u_{m, F E}=-0.0164$ and $u_{m, P E}=0$, respectively.

In the following paragraphs, we will discuss both the superlattices with the electrostatic boundary conditions (figs. 2(a) and 3(a)) and non-electrostatic boundary conditions (figs. 2(b) and 3(b)) at the ferroelectric/ paraelectric interfaces. The case of non-electrostatic boundary conditions can be assumed when the polarization in superlattices aligns parallel to interfaces, the effect of electrostatic coupling between ferroelectric layers, represented by $\frac{1}{2} E_{d, j} p_{j}$, can be ignored. This configuration of in-plane polarization is discussed with the objective of differentiating the effects of interface intermixing and electrostatic coupling.

We first look at the spatial dependence of polarization and internal electric field of the PT/ST superlattices with electrostatic boundary conditions. Figure 2(a) shows the spatial profiles of polarization and internal electric field of $\mathrm{PT} / \mathrm{ST}$ superlattices with $\phi_{F E}=0.5$ (i.e., both thicknesses of PT and ST layers are 3 unit cells) for different values of $\lambda_{0}$. The cases for $\lambda_{0} \neq 0$ indicate the formation of intermixed layers or "dead layers" at $z=0$ [26]. Therefore, the thickness of the intermixed layer is 1 u.c. The

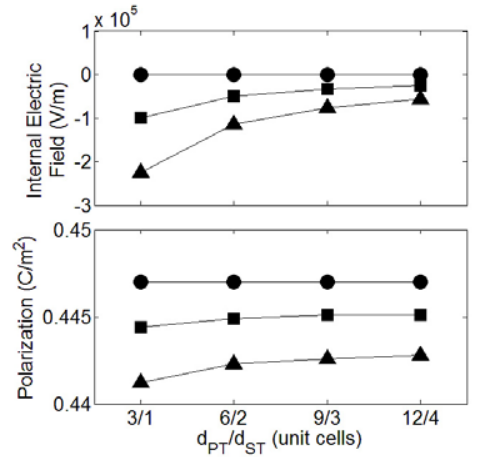

(a)

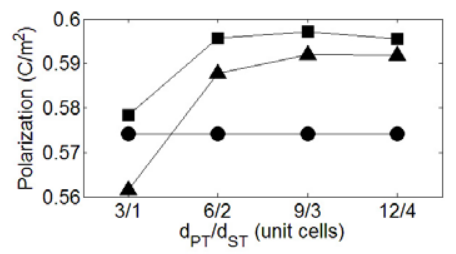

(b)

Fig. 3: Electrical properties at $T=293 \mathrm{~K}$ as a function of $d_{P T} / d_{S T}$ with electrostatic coupling (a) and without electrostatic coupling (b). The values of $\lambda_{0}$ are: $0(\bullet), 0.02 \xi_{0}(\boldsymbol{\square})$ and $\xi_{0}(\boldsymbol{\Delta})$.

existence of the intermixed layer leads to an inhomogeneity of polarization near interfaces, forming the interface region. The spatial dependence of polarization extends into the bulk over a distance governed by its correlation length. It is seen that the continuity or discontinuity of polarization and internal electric field across the interface depends sensitively on the nature of the intermixed layer. The polarization and internal electric field vary spatially in a periodic manner, indicating a periodic interface-induced modulation. As $\lambda_{0}$ increases, both the magnitudes and the gaps of polarization and internal electric field at the interfaces are reduced. Another important features is that the modulated profiles of internal electric field and polarization are correlated. An interesting change of sign in the local internal electric field at the interface region is predicted in a superlattice with $\lambda_{0}=\xi_{0}$ (brown line). The internal field in the PT layer acts as the depolarization field $E_{d, P T}(z)<0$, whereas $E_{d, S T}(z)>0$ tends to enhance the polarization in the ST layer. The internal electric field in the ST layer originates from the electrostatic interaction between polarizations in different PT layers across the ST layer [15]. Hereafter, we denote it as "polarization-induced internal electric field". The magnitude of the depolarization in the PT layer for the superlattice with $\lambda_{0}=0$ is $\left|E_{d, P T}\right| \sim 250 \mathrm{MV} / \mathrm{m} \sim 0.4 E_{C, P T}$ from our calculation, where the bulk coercive field of PT, $E_{C, P T}$, is estimated as $E_{C, P T}=4 \alpha_{P T}^{*}\left(-\alpha_{P T}^{*} / 6 \beta_{P T}^{*}\right)^{1 / 2} / 3 \sim 640 \mathrm{MV} / \mathrm{m}$. Note also that fig. $2(\mathrm{a})$ shows that the internal-field-induced polarization in the ST layer is almost the same as the spontaneous polarization of the $\mathrm{PT}$ layer.

In order to clarify the effect of intermixing at ferroelectric/paraelectric interfaces, we show the modu- 
lation profiles of polarization for $\mathrm{PT} / \mathrm{ST}$ superlattices without considering the electrostatic boundary conditions, as illustrated in fig. 2(b). This configuration with in-plane polarization is analogous to superlattices with interdigital electrodes [31]. In this case, there is no depolarization field in the PT layer and the internal electric field in the ST layer does not exist. If no intermixing occurs, the polarization in the $\mathrm{PT}$ layer is equivalent to its bulk of $P \sim 0.75 \mathrm{C} / \mathrm{m}^{2}$, whereas $P=0$ for the ST layer at $T=298 \mathrm{~K}$, as expected. From figs. 2(a) and (b), it is clearly seen that electrostatic coupling between ferroelectric layers plays a dominant role in enhancing the polarization of superlattices with polarizations aligned perpendicularly to interfaces. Even if no intermixing occurred, the polarizations of both the PT and ST layers are almost similar, as shown in fig. 2(a).

We now investigate the average polarizations $P$ and the internal electric field $E_{d}$ of $\mathrm{PT} / \mathrm{ST}$ superlattices as a function of the unit period with thickness ratio fixed at $d_{F E} / d_{P E}=3$. The average polarization is defined as $P=1 / L\left(\int_{-d_{F E}}^{0} p_{F E} \mathrm{~d} z+\int_{0}^{\mathrm{d}_{P E}} p_{P E} \mathrm{~d} z\right)$ with periodic thickness $L=d_{F E}+d_{P E}$. Similarly, the average internal electric fields is defined as $E_{d}=1 / L\left[\int_{-d_{F E}}^{0} e_{d_{F E}}(z) \mathrm{d} z+\int_{0}^{\mathrm{d}_{P E}} e_{d_{P E}}(z) \mathrm{d} z\right]$. Let us first examine the superlattice with electrostatic coupling, as shown in fig. 3(a). With decreasing periodic thickness from long-period (PT/ST $=12 / 4)$ to short-period $(\mathrm{PT} / \mathrm{ST}=3 / 1)$ structures, both the values of $P$ and $E_{d}$ of a superlattice with $\lambda_{0}=0(\bullet)$ remain almost constant. The internal electric field of the superlattice with $\lambda_{0}=0(\bullet)$ is equal to $E_{d} \sim 0 \mathrm{~V} / \mathrm{m}$, implying that the depolarization field in the PT layer and the internal field in the ST layer compensate each other. For the case of superlattices with $0.02 \xi_{0}(\boldsymbol{\square})$ and $\xi_{0}(\mathbf{\Delta}), P$ decreases with decreasing periodic thickness, whereas the depolarization field $E_{d}$ increases with decreasing periodic thickness. $E_{d} \neq 0$ of superlattices with $\lambda_{0} \neq 0$ is clearly due to the inhomogeneous properties at interfaces as a result of the formation of an intermixed layer at $z=0$, as discussed in fig. 2(a). Figure 3(b) shows $P$ and $E_{d}$ of $\mathrm{PT} / \mathrm{ST}$ superlattices without electrostatic coupling as a function of the unit period. In this case, changes in $P$ and $E_{d}$ are purely due to intermixing at the interface. Without intermixing $\lambda_{0}=0, P \sim 0.575 \mathrm{C} / \mathrm{m}^{2}$ is unaffected by different layer thicknesses, as expected from fig. 3(a). For the case with intermixing $\lambda_{0} \neq 0$, it is seen that $P$ can be tuned by changing the layer thickness. Compared to superlattices with electrostatic coupling (as shown in fig. 3(a)), the change in $P$ is more marked. From figs. 3(a) and (b), it is seen that the electrostatic coupling plays an important role in governing the properties of superlattices with polarizations aligned perpendicularly to interfaces.

Finally, we compare the calculated polarization and transition temperature using our model with electrostatic couplings to those obtained in experiments [21]. Figure 4

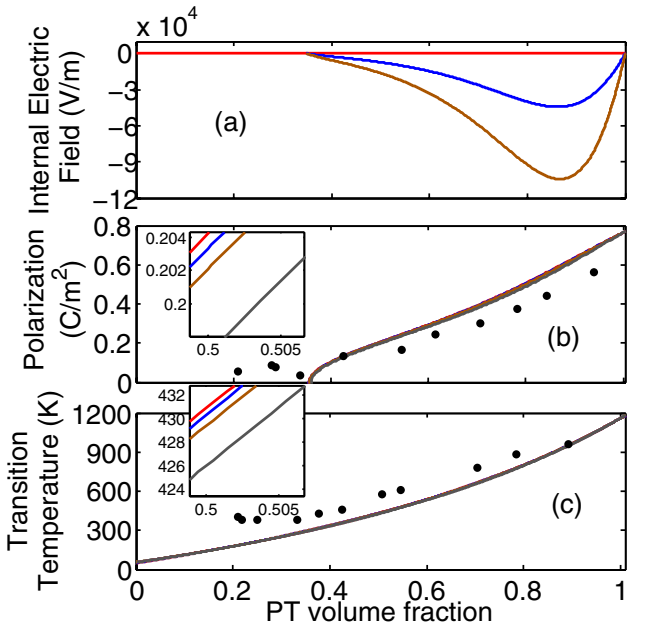

Fig. 4: (Color online) (a) Internal electric field and (b) polarization at $T=298 \mathrm{~K}$, and (c) transition temperature as a function of the PT volume fraction $\phi_{F E}$ of PT/ST superlattices. The values of $\lambda_{0}$ are: 0 (red line), $0.02 \xi_{0}$ (blue line) and $\xi_{0}$ (brown line). Solid dots $(\bullet)$ represent the experimental data. The grey line denotes the result calculated from Dawber et al. [21].

illustrates the average polarization $P$ and internal electric field $E_{d}$ of a PT/ST superlattice as a function of PT volume fraction, $\phi_{F E}=d_{F E} / L$ for various $\lambda_{0}$. Generally, $P$ increases with increasing $\mathrm{PT}$ volume fraction $\phi_{F E}$. For the superlattice with $\lambda_{0}=0$ (red line), $E_{d}=0 \mathrm{~V} / \mathrm{m}$ for all $\mathrm{PT}$ volume fractions $\phi_{F E}$, as expected. For the case of $\lambda_{0} \neq 0, E_{d}<0$ implies that an internal electric field, acting as a depolarization field, exists in the superlattices. It is seen that $E_{d}$ increases with increasing $\phi_{F E}$ until it reaches its maximum value at $\phi_{F E} \sim 0.87$ before dying out (i.e., $\quad E_{d} \sim 0$ ) at $\phi_{F E} \sim 1$. This is expected because PT approaches its bulk at $\phi_{F E} \sim 1$ with the bulk polarization $P \sim 0.75 \mathrm{C} / \mathrm{m}^{2}$. The formation of an intermixed layer at the interface $z=0$ enhances the depolarization field $E_{d}$ (see fig. 4(b)). Polarization $P$ and transition temperature $T_{C}$ (see insets of figs. 4(b) and (c)) of the superlattices are almost unaffected by intermixing at interface, as expected. For comparison, the experimental measurements (solid dots) of polarization and transition temperature are also shown in figs. 4(b) and (c), respectively. It is seen that there is a reasonable agreement between the calculated and measured polarizations for all values of $\lambda_{0}$, indicating that intermixing at interfaces does not have a significant effect on the ferroelectric properties of superlattices (as discussed in figs. 2(a) and 3(a)). We also compare the results with the predictions from the model proposed by Dawber et al. [21]. In the limit of very short periods, the interface effect in PT/ST superlattices is induced by the coupling between antiferrodistortive and ferroelectric instabilities [32]. Since the coupling of structural instabilities at interfaces is not considered, it is reasonable that the model cannot quantitatively capture the experimental data at low volume fraction $\phi_{F E} \lesssim 0.3$. 
In conclusion, we have proposed a thermodynamic model to study electrostatic coupling and interface intermixing in superlattices consisting of alternate layers of ferroelectrics and paraelectrics. We have calculated the internal electric field, polarization and transition temperature, and explained the recently observed polarization and transition temperature in PT/ST superlattices. Our study indicates that intermixing at interfaces forms intermixed layers with properties different from its constituent layers. The formation of an intermixed layer gives rise to inhomogeneous ferroelectric properties in superlattices. The spatial dependence of polarization extends into the constituent layer over a distance governed by its correlation length. We have shown that periodic modulations of the internal electric field and polarization in superlattices are correlated. Intermixing at interfaces has a negligible effect on polarization and transition temperature. The internal electric field, originating from the electrostatic coupling, plays a key role in determining the ferroelectric properties of superlattices.

Ortega et al. studied the dielectric permittivity of $\mathrm{BaTiO} /(\mathrm{Ba}, \mathrm{Sr}) \mathrm{TiO}_{3}$ superlattices [33]. They showed that the dielectric permittivity of the superlattices can be tuned by varying the $\mathrm{Ba} / \mathrm{Sr}$ ratio of the constituent layer without changing the periodicity and the total thickness of the superlattices. This may be another better experimental example of intermixing at interfaces. In this study, the effect of intermixing at ferroelectric/paraelectic interfaces is noticeable only when the electrostatic coupling is neglected when polarizations in the superlattice align parallelly to interfaces. In this case, superlattices with interdigital electrodes [31] may be another practical example for a quantitative correlation between the properties of a superlattice and the degree of the interface effect.

The recent study of local structural distortions in PT/ST superlattices $[20,34]$ revealed the existence of inhomogeneous ferroelectric properties in superlattices. Their studies showed that the theoretical study based on the assumption of uniform polarization throughout the layers in superlattices is most likely not valid $[7,14,15,21,22]$, since a highly inhomogeneous polarization extending over 5-6 unit cells was attributed to ferroelectric domains. Therefore, it is worthwhile to extend the current singledomain model to a multidomain model of intermixing. This work is currently in progress and will be discussed in the near future.

The work was supported by the University of Malaya Research grant (Nos. RG170-11AFR and PV020/2012A). L-HO acknowledges the support from FRGS Grant (203/PFIZIK/6711144) from the Ministry of Higher Learning, Malaysia.

\section{REFERENCES}

[1] Scott J. F., Ferroelectric Memories (Springer-Verlag, Berlin) 2000.

[2] Muralt J., J. Micromech. Microeng., 10 (2000) 136.

[3] Dawber M. et al., Phys. Rev. Lett., 95 (2005) 177601.

[4] Hosokura T. et al., Cryst. Growth Des., 11 (2011) 4253.

[5] Mizoguchi T. et al., Adv. Funct. Mater., 21 (2011) 2258.

[6] Ohnishi T., Koinuma H. and LippmaA M., Appl. Surf. Sci., 252 (2006) 2466.

[7] Pertsev N. A. and Tyunina M., J. Appl. Phys., 109 (2011) 126101.

[8] Fong D. D. et al., Phys. Rev. B, 71 (2005) 144112.

[9] Hung C.-L. et al., J. Appl. Phys., 97 (2005) 034105.

[10] Ishibashi Y., Ohashi N. and Tsurumi T., Jpn. J. Appl. Phys., 39 (2000) 186.

[11] Cooper V. R., Johnston K. and Rabe K., Phys. Rev. $B, 76$ (2007) 020103R.

[12] Shin J. et al., ACS Nano, 4 (2010) 4190.

[13] Qu B. D., Zhong W. L. and Prince R. H., Phys. Rev. $B, 55$ (1997) 11218.

[14] Chew K.-H. et al., Appl. Phys. Lett., 77 (2000) 2755.

[15] Roytburd A. L., Zhong S. and Alpay S. P., Appl. Phys. Lett., 87 (2005) 092902.

[16] Stephanovich V. A., Luk'yanchuk I. A. and Karkut M. G., Phys. Rev. Lett., 94 (2005) 047601.

[17] Pertsev N. A. et al., Phys. Rev. B, 81 (2010) 144118.

[18] Levanyuk A. P. and Misirlioglu I. B., J. Appl. Phys., 110 (2011) 114109.

[19] Chew K.-H., Solid State Phenom., 189 (2012) 145.

[20] Torres-Pardo A. et al., Phys. Rev. Lett., 84 (2011) 220102R.

[21] Dawber M. et al., Adv. Mater., 19 (2007) 4153.

[22] Neaton L. B. and Rabe K. M., Appl. Phys. Lett., 82 (2003) 1586.

[23] Ishibashi Y. and Iwata M., Ferroelectrics, 354 (2007) 8; Chew K.-H. et al., Integr. Ferroelectr., 100 (2008) 79; Chew K.-H., Iwata M. and Shin F. G., Ferroelectr. Lett. Sect., 36 (2009) 12; CHew K.-H., ONG L.-H. and IwATA M., Curr. Appl. Phys., 11 (2011) 755.

[24] Chew K.-H., Ishibashi Y. and Shin F. G., J. Phys. Soc. Jpn., 75 (2006) 064712.

[25] Chew K.-H., Ong L.-H. and Iwata M., J. Appl. Phys., 110 (2011) 054108.

[26] Chew K.-H. et al., J. Phys. Soc. Jpn., 72 (2003) 2364; Tsang C. H. et al., J. Phys. Soc. Jpn., 73 (2004) 3158.

[27] Pertsev N. A., Tagantsev A. K. and Setter N., Phys. Rev. B., 61 (2000) R825.

[28] Stengel M., Vanderbilt D. and Spaldin N. A., Nat. Mat., 8 (2009) 392; ChAng L.-W. et al., Adv. Mater., 21 (2009) 4911.

[29] Landau L. D. and Lifshitz E. M., Electrodynamics of Continuous Media (Elsevier, New York) 1998.

[30] Fong D. D. et al., Science, 304 (2004) 1650.

[31] Harigai T. et al., J. Appl. Phys., 94 (2003) 7923.

[32] Bousquet E. et al., Nature, 452 (2008) 732.

[33] Ortega N. et al., Phys. Rev. B, 83 (2011) 144108.

[34] Zubko P. et al., Nano Lett., 12 (2012) 2846. 